\title{
FPGA Design \& Simulation Modeling of Baseband Data Transmission System
}

\author{
S.Periasamy ${ }^{1}$, Prof. Dr.A.Sivabalan ${ }^{2}$, Prof. Dr.Chandan Madzundar $^{3}$ \\ ${ }^{1}$ Research Scholar, ECE Dept, Allahabad University, \\ ${ }^{2}$ NEC India Ltd, NMNE Center, \\ ${ }^{3}$ ECE Dept, Allahabad University,
}

\begin{abstract}
This paper describes a study on a baseband data transmission system developed for undergraduate students studying communication engineering. Theoretical material, developed in the lectures, is briefly covered. A practical system is presented with pre-detection filtering being employed to improve the bit error rate. A simulation of the complete system is carried out on a Sun work station using the MATLAB simulation package. Simulation and theoretical results are compared.
\end{abstract}

\section{Introduction}

Increases in computing power has provided researchers and educators with a very useful tool, as a current, general purpose PC is powerful enough to run many complex communication and signal processing tasks without expensive, add-on hardware. The implications for engineering education are profound. This paper shows how a communication channel can be modelled using MATLAB and practical, simulated and theoretical results are compared; cementing the students understanding in all three areas simultaneously and resulting in a significant leverage of the students ability compared to tackling the areas separately. In present day usage, the terminology 'data communications' is generally understood to mean the use of encoded digital code streams to allow the communication of information from one digital data device to another. A digital device could be simply a computer, a computer terminal, a high speed printer, a data logging devices or any other type of device whose input or output is a digital data stream. The signal can be directly transmitted over a pair of wires or it can be used to modulate some form of carrier signal which is then sent over an audio, RF, or optical link to the receiving end. The former technique is called baseband signalling and is usually now only used for relatively short distances (less than several kilometres). There are several common means by which the bit stream can be converted into an electrical baseband signal. Here, we are only considering the bipolar non return-to-zero (NRZ) format. However, the system developed can easily be adopted to other formats and students should be encouraged to try to transmit different line codes. In this paper we first introduce the basic theory, discuss the implementation of a practical system, simulate the system using MATLAB, and compare the simulation results with the theoretical results.

\section{Theory}

Consider transmitting a binary data pattern of 0101 (alternate marks and spaces) over a communication channel in the presence of Gaussian noise. Errors may be generated at the receiving end when determining the actual symbols transmitted. The quality of information received is represented by the output bit error rate which is related to the predetection signal to noise ratio (SNR). We wish to evaluate the probability of error at any instant during which

the signal plus noise is sampled.

In the case of NRZ pulse sequences the probability density functions of the signal plus noise and of the noise alone are both Gaussian, see figure 1. Setting a threshold voltage gives rise to the chance of errors occurring, and the probability of an error is given by the area shown by the hatched lines. By inspection, if either symbol, $\mathbf{0}$ or $\mathbf{1}$, is equally likely to be transmitted, then the threshold voltage should be set halfway between the expected signal amplitudes to make the probability of error equal for either case. The probability of error that $\mathbf{1}$ will be mistaken for a $\mathbf{0}$ or vice versa is then:

$$
P_{e}=\int_{V_{T}}^{\infty} \frac{1}{\sqrt{2 \pi N}} \exp \left(-\frac{x^{2}}{2 N}\right) d x
$$




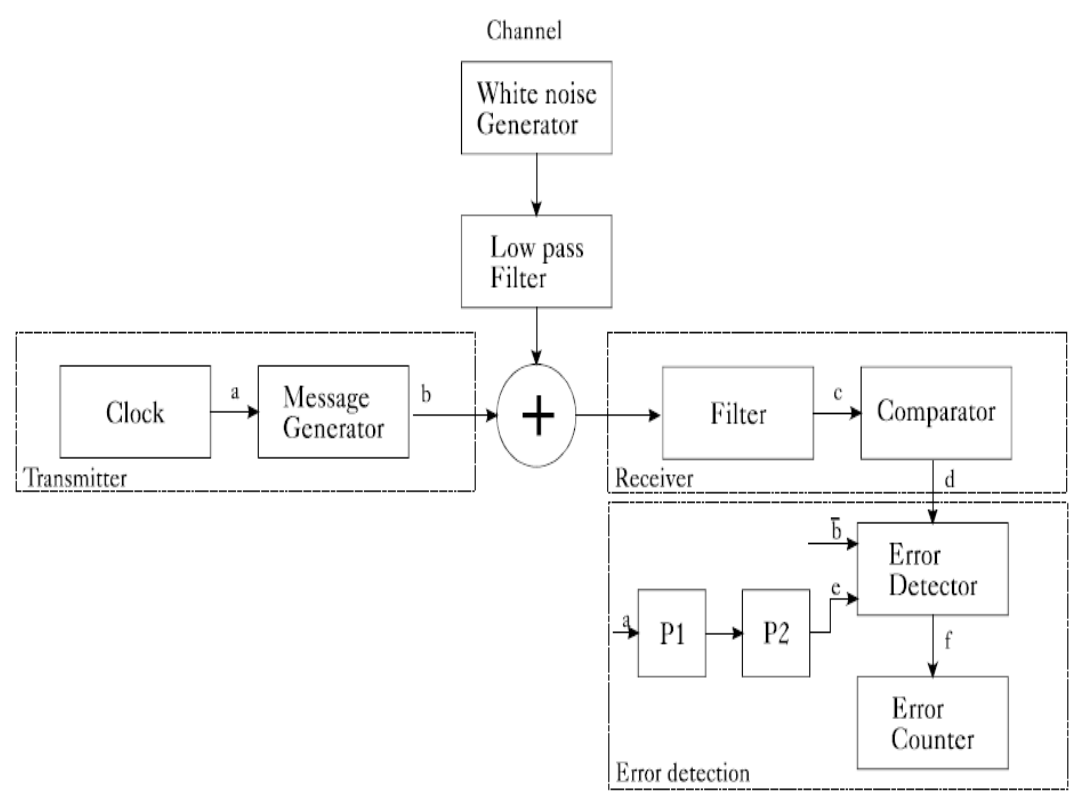

Where $N=$ rms noise power and $x 2=$ mean square value of the received signal amplitude $A$. The integral is not easily evaluated. It is, however, directly related to the error function, tabulated values of which are readily available in mathematical tables. Thus, the probability of error $(P e)$ may be shown as here

$$
P_{e}=0.5 \operatorname{erfc}\left(0.35 \frac{A}{\sqrt{N}}\right)
$$

As can be seen $P e$ is a function of the peak signal voltage $(A)$ and the rms noise power. In many applications $A / \square N$ may not be large enough to provide a tolerable $P e . A / \square N$ also depends on the filter transfer function and signal shape. The particular shape of the received signal is not important. Rather, the question to ask is: for a given signal shape, is it possible to choose a receiver filter transfer function to maximise $A / \square N$ ? The answer is yes, and this is

Done by pre-detection signal processing. The most common technique used is a matched filter just prior to detection. Matched filters compress the energy of the input pulse, which is spread over one bit interval ( $T$ ), so that the amplitude reaches a maximum at one point at $t=T$. Suppose a mark of amplitude $A$ is received in the absence of noise, giving a signal sample $A T$ at the matched filter output (integrated over one bit period). In the presence of noise, the mark signal will be in error if the integrated noise value at the sampling instant is more negative than $-A T / 2$. Following a similar analysis as in $[11,12]$, it can be shown that the probability of error $P e$ for the received unipolar NRZ message using a matched filter is a function solely of the energy in the signal $E=A 2 T$, and the noise power spectral density as given by:

$$
P_{e}=0.5 \operatorname{erfc}\left(0.5 \sqrt{E / \eta_{o}}\right)
$$

Note, that the dependence on the signal shape has been eliminated by use of the matched filter, and the peak $S N R$ is now defined as $S N R=2 E / \square o$. The complications of practical implementation of an ideal integrator suggest an approximation, e.g. a simple $R C$ network with an impulse response of

$$
\begin{aligned}
h(t) & =k \exp (-k t) & 0 \leq t<\infty ; & k=1 / R C \\
& =0 & & \text { elsewhere }
\end{aligned}
$$

To approximate matched filter performance employing linear filters, in this case an $R C$ network, it is essential to optimize the circuit parameter with respect to the input pulse signal. With white noise at the input, the output SNR is given as 


$$
S N R=\frac{2 E}{N_{o}} \cdot \frac{2\left(1-e^{-k T}\right)^{2}}{k T}
$$

Where $N o=4 N / k$. The only parameter that can be optimised is the $R C$ time constant. $S N R$ can be optimised with respect to $k$ by taking the partial derivative of (5), setting the result equal to zero, and solving it using numerical methods. Thus, the optimum time constant is

$$
R C=\frac{1}{k}=\frac{T}{1.26} \quad \text { and } \quad f_{3 d B}=\frac{1.26}{2 \pi T}
$$

Substitution of above SNR equation into RC yields the maximum value of $S N R$ at the filter output:

$$
\left.S N R\right|_{\max }=0.815 \frac{2 E}{N_{o}}
$$

Therefore, the output $S N R$ of the optimised $R C$ filter at $t=T$ is 82 percent of that of the matched filter. This indicates that the performance of the optimised $R C$ filter is only $0.9 \mathrm{~dB}$ below that of the matched filter. The above relationship can also be observed by plotting the deterioration factor $(2(1-e-k T) 2 / k T)$ against $k T$, see figure 2 (without ISI). When the filter bandwidth is less than the optimum value the noise power will be reduced,

but the pulse is distorted and there is significant intersymbol interference (ISI). When the bandwidth is larger than optimum, the output pulse is sharper and ISI is reduced, but more noise is passed. Therefore, a compromise has to be reached between rejecting noise and reducing ISI. The worst case combination of ISI occurs when a data $\mathbf{0}$ follows a $\mathbf{1}$ being transmitted, and is given as $I S I \square \square A e \square k T$. For the optimum value of $k T$ the value of ISI is 0.282 or $-11 \mathrm{~dB}$ relative to the peak value of $A$. This means that with the optimum $k T$, although the maximum value of the SNR is $82 \%$ of that for the matched filter, the ISI can be as much as -11 $\mathrm{dB}$, which is too large. At $-24 \mathrm{~dB}$, then $k T$ is 2.25 (see figure 2) and the $S N R=0.7(2 E / N o$ ), or only $1.49 \mathrm{~dB}$ below that of the matched filter. In order to reduce ISI further to, say, $-30 \mathrm{~dB}$ the $k T$ is 3.43 , and the $S N R=$ $0.568(2 E / N o)$ or $-2.45 \mathrm{~dB}$ relative to that of the matched filter.

The above relationship can also be observed by considering the probability of error, taking into account ISI. This can be showm as:

$$
\frac{E}{N_{o}}=20 \log \left(\frac{\text { measured signal rms }}{\text { noiserms }}\right)+10 \log B_{\text {eq }} T+20 \log \left(\frac{A}{V_{\text {orms }}}\right) d B
$$

By comparing this with the equivalent expression for the matched filter it can be shown that for $k T=$ 2.25 , see figure 2, the $R C$ network will degrade the receiver so that an extra $2.35 \mathrm{~dB}$ of transmitter power is required to achieve the same error rate as that of the matched filter. The ISI can be reduced without significantly degrading the $S N R$ enhancement properties of the optimised $R C$ filter. When the ISI is $20 \mathrm{~dB}$ or more below the output pulse level at $t=n T$, it can be considered negligible.

\section{Structure Operation}

The Diagram shows the basic block diagram of a baseband data transmission system. The transmitter is simply a clock generator, with a frequency of $2 \mathrm{kHz}$, feeding a message generator. The message generator produces a sequence of non-return to zero pulses which is then converted into bipolar signals by using a series capacitor. The channel is simulated with an adder mixing white noise (via a filter of $20 \mathrm{kHz}$ bandwidth) to the data. At the input of the receiver pre-detection filtering is employed to enhance the signal to noise ratio thus reducing the probability of bit error. A comparator is used as a decision making device. At high $S N R$ the comparator will regenerate the transmitted data sequence with negligible probability of error, although there will be some jitter due to the displacement of the data signal edges. However, this is very small and is neglected in this exercise. To measure errors, the original data is then delayed for one bit period, to compensate for the signal delay in the pre-detection filter, before being compared with the output of the comparator. The comparison operation is done by an error detection unit which generates a sampling window and produces an output pulse for every error that occurs within the window. For maximum $S N R$ the sampling pulses must be aligned in time with the peak signal output from the pre-detection filter. An external counter is employed to count the error pulses and the error rate is determined for various signal to noise ratios as measured at the input to the receiver filter. Two types of pre-detection filters are used, a first order $R C$ filter and a matched filter (or correlator 
detector). For the rectangular data pulse shape, the correlation detection simplifies to a reset integrator without the need for multiplication by a local reference sequence. This is realised by using an analogue gate to reset an operational amplifier integrator. The bandwidth of the $R C$ filter is obtained from maximum deterioration factor with ISI (thus minimum $P e$ ) $k T$ is 2.255 , which corresponds to a filter cut-off frequency of $715 \mathrm{~Hz}$. This is best illustrated by plotting $P e$ against filter bandwidth, see figure 7, which shows that for a 1001 repetitive data pattern the desired bandwidth of the filter is $\sim 400 \mathrm{~Hz}$ as expected. However, using this value will result in greater ISI for random data which can be avoided by increasing the bandwidth to around $715 \mathrm{~Hz}$ with very little change in Pe. The error detection unit has three input waveforms, namely, the received data, the initial transmitted data and the sampling pulses which are derived from the clock. Either the data or the sampling sequence may be delayed before error detection. Here, the sampling sequence is delayed using two pulse generators, where P1 sets the required delay and P2 regenerates the sampling pulse width.

\subsection{Hardware Level}

\section{Results}

Students should be asked to measure the rms voltage values of the signal and the noise separately at the input of the receiver filter. Since the pre-detection filter will distort the spectral content and the amplitude of the input signal, it is appropriate to introduce a correction factor in the SNR analysis. Thus, the normalized SNR is given as below

$$
S N R=\left.\frac{E}{N_{o} B_{e q} T} \quad \frac{E}{N_{0}}\right|_{d B}=20 \log \left(\frac{\text { Signalrms }}{\text { Noiserms }}\right)+10 \log \left(B_{e q} T\right)
$$

and the rms voltage at the output of the filter $=$ òTVo rms $T$ Vo $t d t 0$

$$
\frac{E}{N_{o}}=20 \log \left(\frac{\text { measured signal rms }}{\text { noiserms }}\right)+10 \log B_{e q} T+20 \log \left(\frac{A}{V_{\text {orms }}}\right) d B
$$

where $V o(t)$ is the filter output signal. Therefore the energy to noise ratio may be written as:

\subsection{Simulation Level}

The complete system is modelled using the simulation package MATLAB. The advantage of performing a computer simulation of a complex system is that significant changes can be made readily. This allows testing of the system using idealised processing elements which might otherwise take a significant time to design and realise in hardware. In addition, the simulation can support the hardware design by giving optimised component values for crucial parts of the system. It is to be remembered that the hardware system is continuous i.e. all elements of the system are active at once. In the software simulation, code is executed sequentially and discrete data is used. Therefore, blocks of data are processed, one at a time. Data is transmitted around the simulation in vectors and arrays.

\section{Conclusions}

A simple baseband data transmission system has been designed, simulated and built. Simulated results are in agreement with the theoretical predictions. The results indicate that there is $2.351 \mathrm{~dB}$ degradation in $E / N o$ when employing an $R C$ filter as a pre-detection component compared with a matched filter. It was noted that students of average ability gained in awareness of the theory, probably from having to reconcile the theoretical and simulation results. The real benefit however appeared to be for the more able students, who were able to explore the theory and ask "what if" questions which they could then answer using the simulation. Indeed two of the students who did this case study are co-authors of this paper. The advantage of performing a computer simulation is that it allows system designers to make significant changes and try many more approaches in a shorter time than would be possible using real hardware. It also allows one to test the system using idealized processing elements which may take a significant time to design and realize in hardware. Software simulation assumes ideal components with exact values, whereas practical results are subject to component tolerances etc. Finally, the combination of theory, simulation, and laboratory experiments has much to offer when compared to more traditional text based instruction only. 


\section{Simulation Level output Waveform:}

Waveforms of transmitted signal

(a)

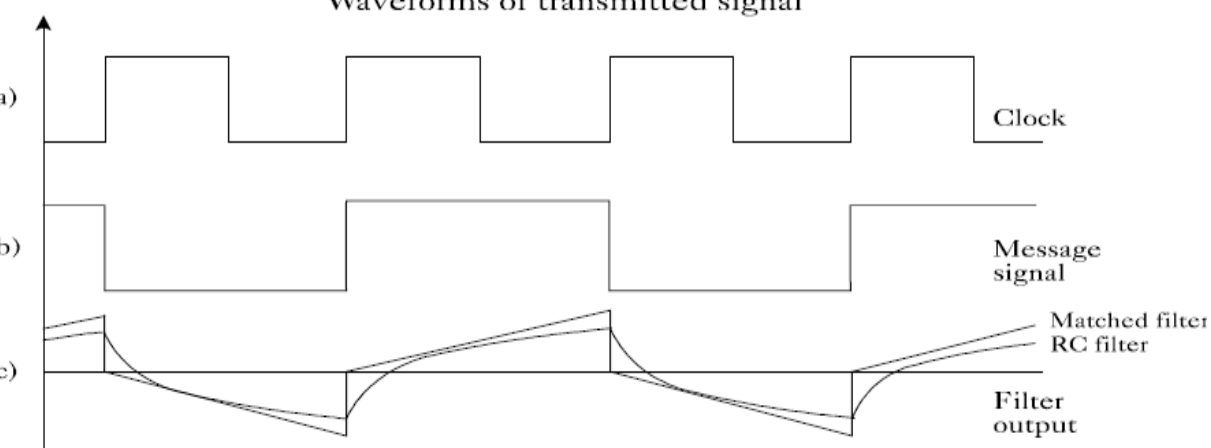

Principle of error detection

(c)

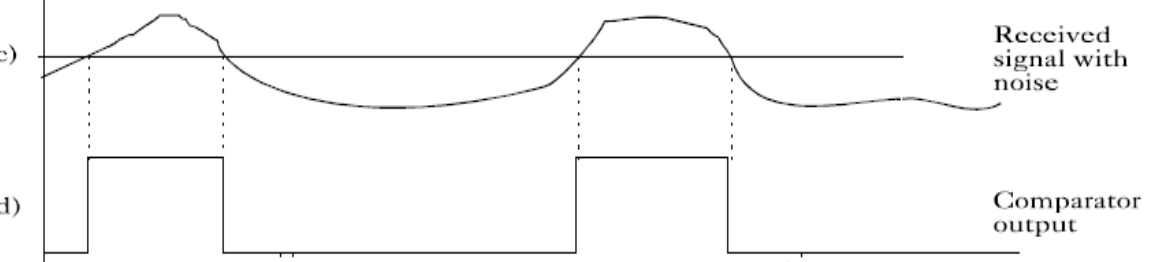

(e)

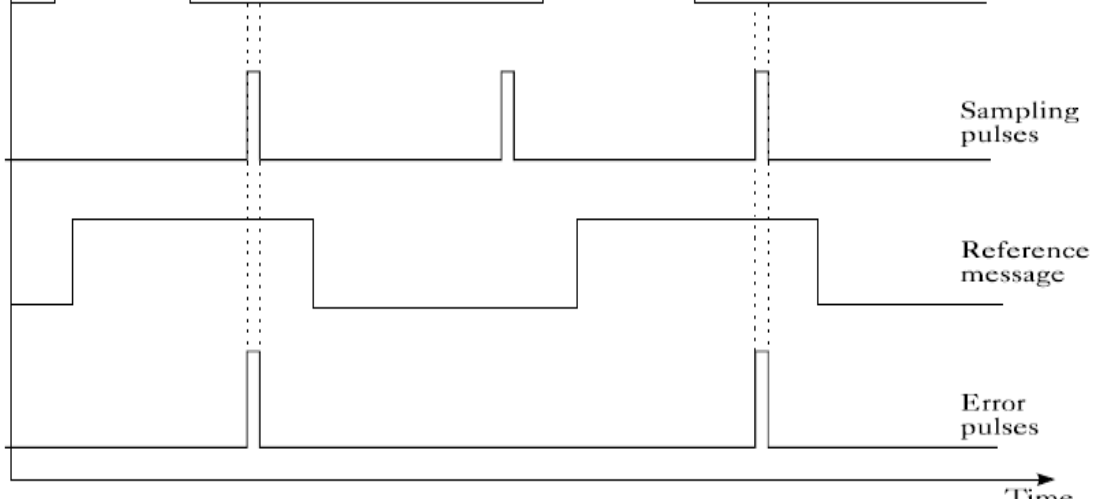

References

[1] Modelling of a Baseband Data Transmission System by Communication Engineering Group, School of Engineering, Sheffield Hallam University,Pond St., Sheffield, UK.2007

[2] Matlab Reference Guide, 1992, The Mathworks Inc.\& Matlab User's Guide, 1992, The Mathworks Inc

[3] Krauss TP, Shure L, Little JN: "Signal Processing Toolbox", 1992, The Mathworks Inc.

[4] King RW: "Speech signal analysis synthesis and recognition exercise using MATLAB”, Int. J. Elect. Eng. Educ., vol. 34, pp- 161$172,1997$.

[5] Medeiro CR, O'Reilly JJ: "Simulation of lightwave systems using COMDISCO", 4 th Bangor Symposium on Communications, 2228 May, pp. 98-101, 1992

[6] Sanmugan KS, et al, "Simulation-based CAD tools for communication and signal processing systems", IEEE Intn. Conf. on Communs., Boston, June 11-14, 1989.

[7] Ahmadi J, et al: "Development of all-digital QSPK modem for mobile satellite application using COSSAP", 4th Bangor Symposium on Communications, 22-28 May, pp. 106-111, 1992.

[8] COSSAP from SYNOPSYS at http://www.synopsys.com/

[9] OPNET Modeler from MIL 3 Inc.: http://www.mil3.com

[10] Ptolemy at http://ptolemy.eecs.berkeley.edu

[11] Schwartz M: "Information Transmission, Modulation, and Noise", McGraw-Hill, $2^{\text {nd }}$ edition, 1970.

[12] Stremler FG: "Introduction to Communication Systems", 3rd edition, chapter 4, 9, 1990,Addison-Wesley Publishing Company, Inc.

[13] Couch LW: "Digital and Analog Communication Systems", 1993, Macmillan Publishing Co., Inc.[14] Matlab code for system at http://homepages.shu.ac.uk/ engajs/matlab.htm. 